\title{
Incoming Flow Lamination Increases the Efficiency of Aerosolized Medication Delivery to 3D Oral Mucosal Tissue Models
}

\author{
G Greg Haroutunian ${ }^{1,2, *}$, Ashot Tsagikian ${ }^{3}$, Elena Fedorova ${ }^{4}$, Haiyan Zheng ${ }^{5}$, G Luca Gusella ${ }^{4}$ and Arevik Mosoian ${ }^{4, *}$ \\ ${ }^{1}$ Keck School of Medicine, University of Southern California, Children's Hospital Los Angeles, California, USA \\ ${ }^{2}$ AltaMed Health Services, California, USA \\ ${ }^{3}$ Data Processing and Field Engineering Corp., Glendale, California, USA \\ ${ }^{4}$ SkinAxis LLC, Cedar Knolls, New Jersey, USA \\ ${ }^{5}$ Rutgers University, Piscataway, New Jersey, USA
}

*Corresponding authors: G Greg Haroutunian, Keck School of Medicine, University of Southern California, Children's Hospital Los Angeles, California, USA, E-mail: gregharoutunian@yahoo.com

Arevik Mosoian, SkinAxis LLC, Cedar Knolls, New Jersey, USA, E-mail: arevik.mosoian@skinaxis.com

Received: 14 Jun, 2021 | Accepted: 30 Jun, 2021 | Published: 13 Jul, 2021

Citation: Haroutunian GG, Tsagikian A, Fedorova E, Zheng H, Gusella L, et al. (2021) Incoming Flow Lamination Increases the Efficiency of Aerosolized Medication Delivery to 3D Oral Mucosal Tissue Models. Int J Dent Oral Health 7(5): dx.doi.org/10.16966/2378-7090.372

Copyright: @ 2021 Haroutunian GG, et al. This is an open-access article distributed under the terms of the Creative Commons Attribution License, which permits unrestricted use, distribution, and reproduction in any medium, provided the original author and source are credited.

\begin{abstract}
Background: Inhalable medication devices on the market deliver aerosolized drugs in a turbulent flow, which in complex interaction with oropharyngeal geometry causes the major portion of the drug to deposit locally, while creating significant obstacles for reaching the lower lungs. The unintended deposition of aerosolized medications in the oropharynx is known to have negative effects on oral health. The emergence of numerous new aerosolized medications on the market is very likely to add significantly to the list of side effects. We hypothesized that lamination of the outflow by sequentially subdividing the aerosol flow within the spacer into smaller sub-flows using internal septi of different lengths will improve the delivery of the aerosol to distant targets while reducing its deposition in anterior aspects of the airways, especially in oropharyngeal cavity.

Methods: Here we compared the efficiency of aerosolized medication (fluticasone) delivery via a Standard Spacer (SS) to the novel Flow Modification Device (ModiFlow) by using oral mucosal 3D tissue culture (SkinAxis) as a target for drug delivery. ModiFlow is a spacer-like cylindrical device within which a system of septi of different lengths sequentially subdivides the flow while keeping its cross-sectional area constant. The efficiency of fluticasone delivery to the surface of tissue cultures was quantified by mass spectrometry.

Results: The results of the study demonstrated a statistically significant advantage of Modiflow over a Standard Spacer in delivering aerosolized fluticasone to target tissue at different distances, which was more pronounced at a longer distance.
\end{abstract}

Keywords: Spacer; Three-dimensional oral mucosal tissue; Fluticasone deposition; Mass spectrometry; Turbulence

Abbreviations: MF: ModiFlow; SS: Standard Spacer; 3D: Three Dimensional oral mucosal tissue models; TT: Test Tubes

\section{Introduction}

In recent years the targeted aerosol delivery to the respiratory tract has rapidly gained interest as the preferred route for the treatment of lung diseases locally, as well as accessing the systemic circulation. Many therapeutic aerosols containing large molecules-proteins, hormones, nucleic acids, chemotherapeutic drugs $[1,2]$, have shown promise as agents for gene therapy, antiviral therapy for influenza and measles, insulin and vaccine delivery $[3,4]$.

Our increasing understanding of medication receptor distribution throughout the lungs further emphasizes the need for delivering different aerosolized medications to very specific areas of the respiratory tract [5]. In recent years more than 100 different medications have been brought to the market in aerosolized form [6]. However, the efficiency of aerosolized medication delivery to the lungs remains extremely low due to significant variability in parameters within the upper respiratory tract and the design of the delivery devices. Consequently, up to $77 \%$ of the medication can deposit in the upper airways [7], most notably in the oral and pharyngeal areas, thus not only reducing efficiency and lowering the predictability of the outcomes but also causing side effects. Deposition of large amounts of medication in the oral cavity poses a significant danger to health. The known local side effects from just a few asthma medications include xerostomia, dental caries, candidiasis, ulceration, gingivitis, periodontitis, taste changes, and others [8,9]. Systemic ones are well known and include adrenal suppression, short stature, and others. It is to be expected that the exponential growth in the number of aerosolized drugs with a much wider spectrum of bio-activity poses new and unpredictable dangers to oral health. Therefore, it is imperative to significantly reduce the deposition of aerosolized drugs 
in the mouth and throat, and at the same time to improve their delivery to the lower lungs. The interaction of the aerosolized medication outflow from a delivery device with the complex and irregular geometry of the oropharyngeal cavity produces a very turbulent flow. The emergence of turbulence is one of the main reasons for high oropharyngeal deposition rates. Even a small reduction in turbulence at this first segment of the shared space within the respiratory tract may offer a significant opportunity for not only decreasing the amount of medication getting deposited in oropharyngeal cavity but also for improved further propagation of the aerosol into the respiratory tract. Of the many parameters involved in aerosol administration, one that we identified as amenable to manipulation is the structure of the aerosol outflow from the delivery device. To date, very little attention has been paid to the laminarity of the outflow in any of the devices on the market, including the one representing the best clinical standard that combines pressurized Metered Dose Inhaler (pMDI) with an attached Spacer. With the entrance of newer inhalable medications to the market, new delivery standards are being explored $[10,11]$.

The emergence of turbulent flow in different segments of the respiratory tract can have a significant impact on the deposition and absorption of aerosols. For many aerosolized medications, this may be a factor of critical importance. The presence of turbulence can increase the localized shear wall stress [12], create significant resistance to the flow, and reduce further propagation of the aerosolized substances. The turbulence emerging from the delivery device itself as a possible cause for high rates of oropharyngeal deposition and obstacle to the effective delivery of aerosols to the lungs has not been elucidated. We thought that lamination of the device outflow (with a presumed reduction of turbulence) to optimize its interaction with oropharyngeal inspiratory airflow, may have a positive impact on the efficiency of aerosol delivery. Even with the use of spacers, despite the variations of "external" parameters to improve their performance, except for volume, the overall efficiency of aerosol delivery to the lower lungs remains poor [13-15]. The inner space of a spacer device, where the flow of aerosolized medication occurs, offers a significant opportunity for manipulating the flow [16].

Most, if not all of the existing spacers on the market lack any internal structures, and the exiting flow of aerosol is highly turbulent $[17,18]$. A device that can reduce the turbulence in the aerosolized medication outflow may prove to be more efficient in delivering the medication to the lower lungs and reducing its deposition in the upper airways [16]. We hypothesized that lamination of the outflow by sequentially subdividing the aerosol flow within the spacer into smaller sub-flows using internal septi of different lengths will improve the delivery of the aerosol to distant targets while reducing its deposition in near ones.

ModiFlow (MF) is a novel spacer-like delivery device specifically designed to create a Laminar Outflow of aerosolized medication by sequentially subdividing the flow by a system of internal septi of different lengths while keeping the total cross-sectional area constant (Flow Modification Device, G. Greg Haroutunian, MD, US Patent \# $8,371,291$ B2). Such a "strategy", which is characterized in terms of fractal geometry, is widely used in biological delivery systems, such as the bronchial tree, vascular system, others. Sequential division (bifurcation) of the flow while keeping the total cross-section area relatively constant along the flow path is thought to minimize the emergence of turbulence, and therefore improve the delivery of the fluid or gas [19].

Modeling of the airflow and particle deposition rates in the airways is often done with the use of Computational Flow Dynamics (CFD) methods [20-22]. However, the extreme complexity of parameters and factors to be accounted for limit the capabilities of these methods. More direct methods of assessing the medication deposition and absorption rates could be obtained on actual 3D tissue samples. In recent years progress has been made in constructing 3D tissue models and using them for various research and clinical applications [23-26]. This allowed for departure from the use of animal tissue models, and for obtaining data in realistic physiologic conditions [26-32].

\section{Materials and Methods}

\section{Aerosol drug delivery devices}

In this study, we compared the performance of the ModiFlow (MF) to an idealized Standard Spacer (SS). For standardization purposes, a hollow cylindrical tube of identical length and inner diameter represented both devices, with the only difference being that MF had specifically designed inner septal structures, which SS didn't.

Since most, if not all spacer devices on the market have a cylindrical shape, and no internal structures, our Standard Spacer model was considered to be a fair representation of spacers on the market. In addition, several studies have demonstrated that with the exception of volume, all other structural modifications to the spacers, such as valves and masks, have not made a significant difference in their performance [33]. Our goal was to minimize any variability between the tubes for the purpose of controlling all parameters, and leaving only one main difference-the presence of inner septal structures in MF, and the absence thereof in SS.

The parameters for ModiFlow were selected as follows: Total length $-100 \mathrm{~mm}$, Inner Diameter $-38 \mathrm{~mm}$, Septi $-62 \mathrm{~mm}, 34 \mathrm{~mm}$, and $24 \mathrm{~mm}$. The calculated Inner Surface Area (ISA) was $164.9 \mathrm{sq} . \mathrm{cm}$, of which 45.6 sq. $\mathrm{cm}$ was accounted for Septi only, and 119.3 sq. $\mathrm{cm}$ for inner walls. As a SS, a hollow cylindrical tube of the same length $(100 \mathrm{~mm})$ and Inner Diameter $(38 \mathrm{~mm})$ was used, with the calculated ISA of 119.3 sq. $\mathrm{cm}$. No inner septi were present in SS.

To compare the efficiencies of aerosol delivery for MF $v s$ a SS, which was defined as the amount of aerosolized medication, delivered to the target surface area from a pre-defined total amount sprayed into each tube. Four different Test Tubes (TT) have been devised, all of which had a similar structure as depicted in figure 1 . Two of them had a length of $15 \mathrm{~cm}$ and two $-30 \mathrm{~cm}$. All four TT's were of cylindrical shape and had the same inner diameter of $38 \mathrm{~mm}$. In each TT's either MF (Figure 1.5 with spacer 3 ) or a Standard Spacer (Figure 1.5 without spacer 3) was inserted at proximal end (Figure 1.4). The proximal end of TT's was also furnished with an adapter (Figure 1.4) for the Medication Pump (Figure 1.5 without spacer 3), while the distal end with a receptacle (Figure 1.1) for tissue culture. The distances from the exit of the spacer (MF or SS) to the distal end of TT (where the tissue culture was placed) to be either $5 \mathrm{~cm}(15 \mathrm{~cm}-10 \mathrm{~cm}=5 \mathrm{~cm})$ or $20 \mathrm{~cm}$ $(30 \mathrm{~cm}-10 \mathrm{~cm}=20 \mathrm{~cm})$, thus creating four different designs of TT: MF-5, MF-20, SS-5, SS-20. These distances from the spacer exit to the tissue culture were selected to be either "within" $(5 \mathrm{~cm})$ or "outside" $(20 \mathrm{~cm})$ of the average length of adult oropharyngeal cavity-11.5-12.5 cm [3436] (This would allow for four pairs of comparisons to be made: MF- 5 to SS-5, MF- 5 to MF-20, MF-20 to SS-20, and SS- 5 to SS-20. The total Internal Surface Area (ISA) for each Test Tube was respectively MF5-224.6 sq. cm, MF-20-403.5 sq. cm, SS-5-179 sq. cm, SS-20-358 sq. $\mathrm{cm}$, excluding the cross-sectional surface areas (tissue cultures and receptacles).

\section{Aerosolized medication}

Fluticasone Propionate Metered Dose Inhaler (220 $\mu \mathrm{g}$ per spray) was used in all test tubes. 


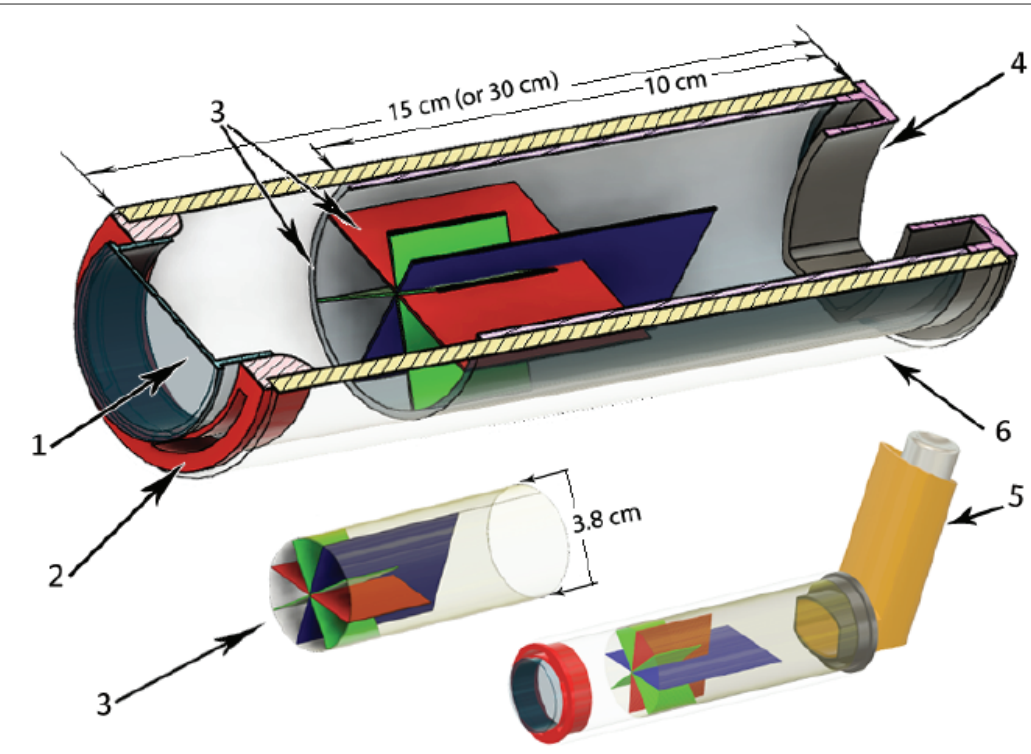

Figure 1: ModiFlow design with expanded view of the septated spacer (top). Total length: $100 \mathrm{~mm}$. Septi: $62 \mathrm{~mm}, 34 \mathrm{~mm}, 24 \mathrm{~mm}$. The main components of the device are indicated: 1) Exit (cell target membrane); 2) Membrane holder; 3) Septi; 4) Medication pump adapter; 5) Medication pump; 6) Spacer tube.

\section{D oral epidermal tissues}

To better approximate physiological conditions of drug delivery, we measured aerosolized drug deposition on a SkinAxis model of the oral mucosal tissues. Normal human Gingival keratinocytes (SkinAxis) were cultured on specially prepared cell culture inserts using serumfree medium and differentiated in vitro using proprietary SkinAxis' cell culture technology to form multilayered, highly differentiated models of the human gingival phenotypes (Figure 2). SkinAxis oral epidermal tissue models are highly reproducible and exhibit in vivo like morphological and growth characteristics. The differentiated tissue was inserted at the end of the spacer, as described above and to quantify drug deposition tissues were processed for Mass Spectrometry.

\section{Fluticasone extraction from oral tissues and quantification}

Each tissue sample was processed by: adding $50 \mu \mathrm{l} 0.1 \%$ formic acid and $200 \mu \mathrm{l}$ of methanol to a culture plate, scrapping with pipette tips, and transferring to an Eppendorf tube. The plate was washed sequentially with $200 \mu \mathrm{l} 0.2 \%$ formic acid and $100 \mu \mathrm{l}$ methanol and the washes were combined with the initial extract. Extracts were sonicated for $1 \mathrm{~min}$ and centrifuged for $5 \mathrm{~min}$ at $25000 \times \mathrm{g}$.

Supernatants were diluted 10 -fold using $50 \%$ methanol/ $0.1 \%$ formic acid before analysis by LC-MS.

HPLC-MS experiments were performed using a Thermo Fisher Velos LTQ Orbitrap Pro mass spectrometer interfaced with a Dionex U3000 chromatography system. Samples $(5 \mu \mathrm{L})$ were injected in microliter pick-up mode and separated on a reverse-phase column (Discovery BIO Wide Pore C18, $5 \mathrm{~cm} \times 2.1 \mathrm{~mm}$, Supelco Analytical). Chromatography was conducted at a flow rate of $200 \mu \mathrm{l} / \mathrm{min}$ using a gradient formed with an aqueous solution of $0.2 \%$ acetic acid (solvent A) and methanol (solvent B) as follows: 60\% B (1 min), 60-90\% B (linear increase in $3 \mathrm{~min}$ ), $90 \% \mathrm{~B}$ for $1 \mathrm{~min}, 90-60 \% \mathrm{~B}$ (linear decrease in 0.1 $\mathrm{min})$, and equilibration at $60 \% \mathrm{~B}(3 \mathrm{~min})$. The column temperature was maintained at $45^{\circ} \mathrm{C}$. MS acquisition parameters were as follows: the electrospray ion source was operated in positive ion mode (ESI+). The positively charged fluticasone $(\mathrm{m} / \mathrm{z}=501.3)$ was isolated in the

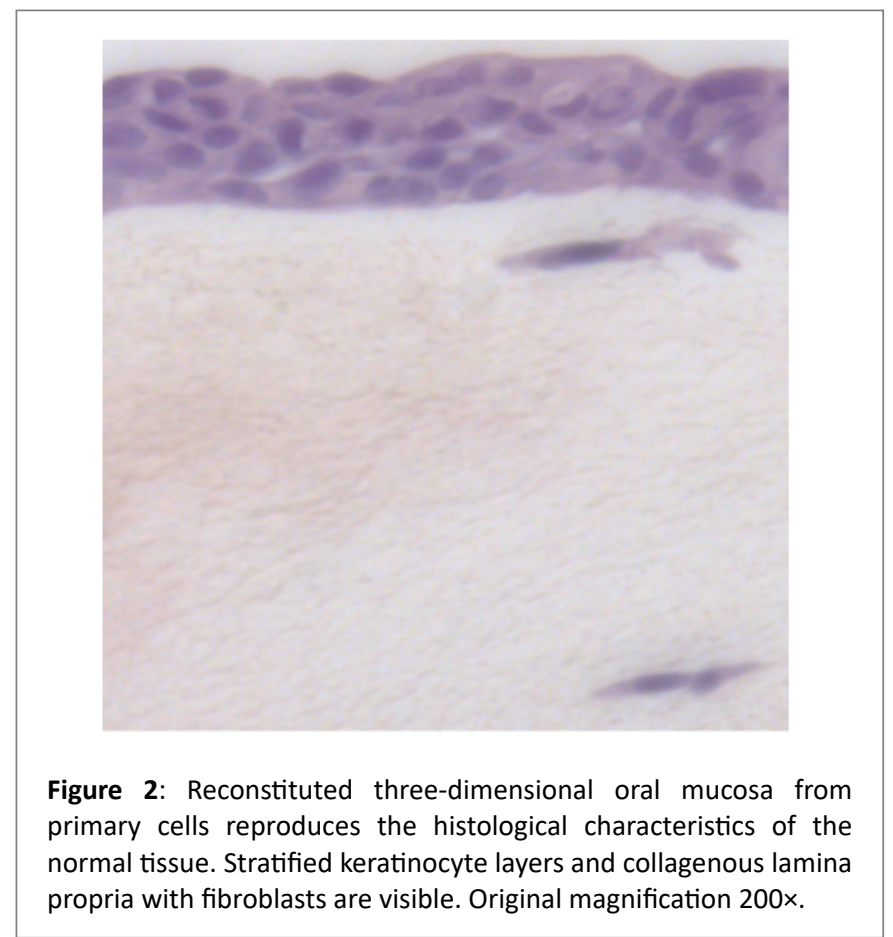

ion trap with an isolation window of $3 \mathrm{~m} / \mathrm{z}$ and fragmented with CID with a relative collision energy of $25 \%$ and activation time of 10 milliseconds. Fragments were detected using the ion trap and the $303.15 \mathrm{~m} / \mathrm{z}$ fragments used for quantification.

A standard curve consisting of dilutions of fluticasone in methanol $(0.01 \mathrm{ng} / \mathrm{ml}$ to $100 \mu \mathrm{g} / \mathrm{ml})$ was analyzed in parallel with samples. Peak areas of the 501-301 transitions were measured using XCalibur software. Concentrations of fluticasone in samples were determined with respect to standard curves by non-linear regression using a fourparameter sigmoidal fit, weighted by $1 / \mathrm{y}^{\wedge} 2$. 


\section{Statistical analysis}

For each of the two spacers, independent $t$-tests were used to test the effects of varying drug deposition in different experimental settings. P-value $<0.05$ was considered statistically significant.

\section{Results}

\section{Comparing the efficacy of drug delivery of Standard Spacer to ModiFlow}

The geometry of ModiFlow inner space is structured using uniquely designed longitudinal septi of different lengths that produce laminated flow, which persists after exiting the device. Subdivision of the main flow into two sub-lows, a process that is subsequently iterated multiple times, while keeping the total cross-section area on all levels of subdivision constant, helps to disrupt the cycle of growth of the lateral force, preventing the emergence of a high Reynolds number turbulence. This tree-like branching pattern (fractal tree) generated by iteratively applying a set of simple rules is pervasive in biological networks. It is also utilized to model lung function and the bronchial tree [37]. In ModiFlow the septi serve to prevent the emergence of turbulence by periodically sub-dividing the flow, and with it, dividing the lateral (tangential) force that grows in an iterative side-to-side movement to eventually become strong enough to break a laminar flow into a turbulent one.

This lamination of the flow can have the following potential implications: 1) it can potentially prevent the emergence of high Reynolds number turbulence; 2) a higher likelihood of such a laminated flow to overcome the oropharyngeal barrier without depositing most of the particles there, a larger portion of the flow may reach trachea and main bronchi, and skew the distribution of aerosol particles pattern towards deposition in lower parts of the respiratory tract, including finer bronchioli and alveoli; 3) manipulation of the geometry of ModiFlow could allow a more targeted deposition of particles in specific areas of respiratory tract depending on the need and type of medication being delivered and the distribution map of its receptors in the airways; 4) reduction of side effects due to reduced deposition in the mouth, throat, trachea and upper bronchi; 5) improved/more efficient delivery may overall reduce the cost of expensive medications.

The tissue samples were inserted at the distal end of each Test Tube (TT) through an adaptor (Figure 1, membrane holder), perpendicular to the axis of the TT (axis of flow), opposite to the end where the medication dispenser was inserted. ModiFlow or Standard Spacer were incorporated flush with the proximal end of the TT, and oriented in such a way that the flow is directed towards the tissue sample (Figure 1). The positioning of the tissue sample perpendicular to the axis of flow at different distances allows for the assessment of aerosol flow (delivery) through a particular cross-section of the TT. Placing the tissue samples on the lateral walls of the TT would make it very difficult to discern any correlation between the aerosol flow and its deposition on the tissue sample, having to take into account too many variables.

Experiments were conducted to maximally mimic the real-life application of aerosol oral delivery of Fluticasone. After shaking for 5 seconds and spraying away once to assure proper functioning, the Fluticasone Metered Dose Inhaler was inserted into the medication pump adapter of the Test Tube containing the target tissue and was activated twice with a 3-minute interval by pushing the top of the medication canister all the way down. This delivered a total dose of $440 \mu \mathrm{g}(220 \mu \mathrm{g}$ per spray) in accordance with standard dosing recommendations. Because of the hermetic closure of Test Tubes, and the same standard amount of Fluticasone delivered into each TT, it was possible to not only measure the amount of Fluticasone delivered along the axis of flow to the tissue samples at different distances but to also indirectly assess the amount of Fluticasone deposited on the lateral walls of each TT. This could be achieved by subtracting the amount of Fluticasone deposited on each tissue sample from the total dose of $440 \mu \mathrm{g}$ sprayed in each TT.

To provide consistent aerosol droplet sizes and flow velocities, as well as equal doses of Fluticasone, and to control for these variables, we used the same Fluticasone MDI in all Test Tubes. To minimize possible electro-static influences, all Test Tubes and spacers were pre-washed before each experiment with standard dishwasher detergent.

The results presented in figure 3 indicate that ModiFlow had significantly increased drug delivery to the tissue as compared to the Standard Spacer. ModiFlow delivered on average $20.19 \pm 5.2 \mu \mathrm{g}$ and $8.9 \pm 4.1 \mu \mathrm{g}$ of drug/tissue at $5 \mathrm{~cm}$ and $20 \mathrm{~cm}$ distance, while Standard Spacer delivered $7.8 \pm 0.8 \mu \mathrm{g}$ and $3.05 \pm 0.9 \mu \mathrm{g} /$ tissue respectively (Figure 3), strongly suggesting a more efficient Fluticasone delivery to mucosal tissue targets by ModiFlow.

\section{Discussion}

The efficient delivery of aerosolized medications to the patient's airways continues to be a challenging task. Various devices such as pumps, spacers, discs and nebulizers are currently used for that purpose. In all of the devices on the market, the outflow of aerosolized medication is presumed to be significantly turbulent. Turbulent flow is one of the reasons for the deposition of up to $77 \%$ of the medication in the upper airways $[7,12]$. With the advent of numerous new aerosolized medications with strong biological activity entering the marketplace, and with the aerosol delivery technology still significantly lagging in efficiency, the deposition of large amounts of such medications in oropharyngeal cavity is increasingly becoming a cause for concern for numerous potential dangers posed by them to oral health. The interaction of the outflow of a delivery device with the complex geometry of the oropharyngeal segment of the upper airways may further contribute to these inefficiencies. To our knowledge, no specific measures have been taken so far to modify the outflow of these devices with the intention to eliminate, reduce, or in some other manner alter the outflow turbulence. Accurately assessing the efficiency of laminated aerosol delivery may yield important results.

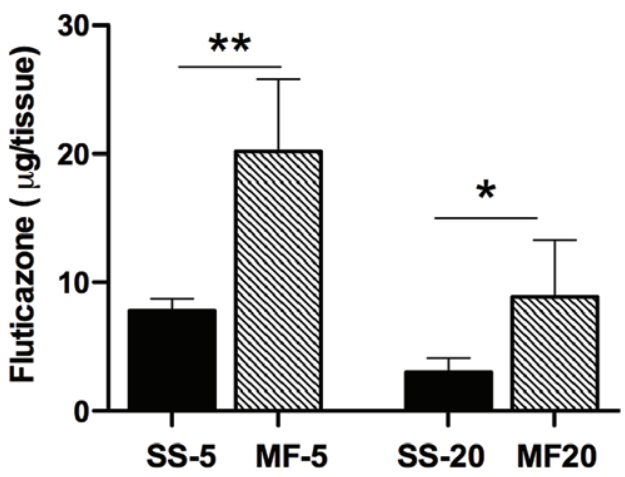

Figure 3: Different delivery efficiencies of Fluticasone to 3D oral mucosa tissue models. Absorption of aerosolized Fluticasone on live tissues upon delivery with Standard Spacer (SS) and ModiFlow (MF) at $5 \mathrm{~cm}$ and $20 \mathrm{~cm}$ distance from spraying device. ${ }^{*} \mathrm{p}<0.05 ;{ }^{*} \mathrm{p}<0.01$ by $t$ test. 
It is important to note that all 4 Test Tubes with attached medication pumps constituted a closed system, and the amount of Fluticasone sprayed into each one of them was identical. Although both the ModiFlow and the Standard Spacer have the same length and diameter, the Inner Surface Area of the Standard Spacer is 119.3 sq. $\mathrm{cm}$, while that of the ModiFlow is $38 \%$ larger- 164.9 sq. $\mathrm{cm}$ due to the presence of inner septi. This would cause a larger amount of Fluticasone deposition within ModiFlow as compared to the Standard Spacer. Since the amount of Fluticasone that reached the cell cultures at the end of Test Tubes was significantly higher (2.5-3-fold) with ModiFlow than with Standard Spacer, therefore smaller amounts were deposited elsewhere in Test Tubes with ModiFlow. As indicated above, the delivery of the aerosolized Fluticasone as measured by the tissue surface deposition rates was significantly more effective via ModiFlow than via Standard Spacer in both distance ranges-short and long $(\mathrm{p}<0.01$ and $\mathrm{p}<0.05$ respectively). Interestingly, the comparative efficiency of ModiFlow can be judged to be higher in the long-range than in the short-range if ratios of deposited Fluticasone are used: $8.89 / 3.05=2.91$ (long-range) $>20.18 / 7.79=2.59$ (short-range). This may be due to 2 different factors: 1 . The difference in the lengths of Laminar Outflow between MF and SS is more pronounced in longer Test Tubes, giving the laminated outflow of ModiFlow more time to exert its 'positive effects on the efficiency of delivery. 2. The share of ISA of Septi in Total Test Tube ISA is almost twice lower in longer Test Tubes with MF $(45.6 / 403.5=0.113)$ than in shorter ones $(45.6 / 224.6=0.203)$.

From here it may be deduced, with some level of certainty, that not only higher amounts of Fluticasone are being delivered to target tissues via ModiFlow compared to Standard Spacer, but also smaller amounts of it are being deposited on the walls of the Test Tube despite the higher ISA in ModiFlow.

If the advantages of ModiFlow in improving the aerosolized medication delivery are confirmed in realistic airway models, as well as with clinical outcomes, bringing this device to clinical practice could benefit the field of drug delivery. Moreover, future studies may help fine-tune the device for the purpose of controlling the degrees of flow lamination and turbulence reduction, which in turn can offer a tool for targeting the delivery of a specific drug to a specific area of airways/lungs, or systemic circulation. The simple design and the wide range of possibilities for tweaking its geometry without violating its basic structure allow for the easy manufacturing of a large number of ModiFlow versions customized for different drugs, targets, patient ages and sizes, etc.

\section{Conclusion and Perspectives}

Turbulence plays a significant role in reducing the efficiency of aerosolized medication delivery to the lungs. In the ModiFlow device, the inner space is structured in a manner that helps laminate the outflow and consequently reduces the emergence of turbulence in the oropharyngeal cavity. Evaluation of aerosolized drug delivery using real-world exposure scenarios could provide a better interpretation and the assessment of the efficiency of drug delivery. However, most of the current in vitro studies do not reflect realistic conditions of human tissue exposure to aerosolized medication delivery due to different variables (cultured conditions, primary cells derived from different donor variations). In this work, we studied the impact of repeated exposure of fluticasone on a three-dimensional (3D) reconstructed human oral tissue derived from a single donor, using a nebulizer system focusing on drug deposition effects.
This study demonstrated that despite the larger inner surface area, ModiFlow improves the efficiency of aerosol delivery in comparison with an idealized Standard Spacer as measured by the amount of aerosolized Fluticasone delivery to 3D tissue cultures placed perpendicularly to the axis of the flow.

The difference in efficiency was more pronounced when the target tissues were placed at farther distances. The results of the study have also indirectly indicated that ModiFlow reduces the aerosol deposition on the lateral walls of Test Tubes when compared with a Standard Spacer. It is reasonable to speculate that these advantages of outflow lamination with the use of ModiFlow may prove to be also present when realistic airway models are used instead of cylindrical Test Tubes, which would be a subject for a future study. As the need for efficient, consistent and targeted delivery becomes increasingly important with newer aerosolized medications entering the marketplace with high potential for side effects or high cost of medication waste, all factors that may have a role in that process will need to be explored and validated. Manipulation of the flow structure using the inner space of a spacer device, alteration of the outflow, and consequently the manner in which it interacts with the inspiratory flow of the oropharynx, possible reduction of flow turbulence-these are all potential points of application for achieving our goals.

ModiFlow offers an opportunity to structure the aerosol flow within its space in a manner that can significantly alter the interaction of that flow with the complex geometry of the airways. This may have a positive effect on the reduction of undesired side effects in the oral cavity. Moreover, the geometry of the device's inner structure can theoretically be customized for different drugs, lung targets, patient ages and sizes.

\section{Data Availability Statement}

All the data used to support the findings of this study are included within the article.

\section{Funding Information}

This work was supported by funding from SkinAxis LLC.

\section{Author Contributions}

All authors contributed to data collection, data analysis, and interpretation, drafting and revising the article, and approved the final manuscript. G. Haroutunian conceived the hypothesis, A. Mosoian, E Fedorova and L. Gusella obtained the funding. A. Mosoian and G. Haroutunian taking responsibility for the integrity of the work. G. Haroutunian, A. Mosoian, E Fedorova, and L. Gusella designed the study protocol. A. Mosoian, E Fedorova, A Tsagikian, and H Zheng guided the execution of the experiments.

\section{Competing Interests}

G. Greg Haroutunian is the inventor of ModiFlow (US Patent \# $8,371,291 \mathrm{~B} 2)$.

All other authors declare that they have no competing interests.

\section{References}

1. De Benedictis FM, Selvaggio D (2003) Use of inhaler devices in pediatric asthma. Paediatr Drugs 5: 629-638.

2. Kleinstreuer C, Shi H, Zhang Z (2007) Computational analyses of a pressurized metered dose inhaler and a new drug-aerosol targeting methodology. J Aerosol Med 20: 294-309. 
3. Kleinstreuer C, Zhang Z, Donohue JF (2008) Targeted drug-aerosol delivery in the human respiratory system. Annu Rev Biomed Eng 10: 195-220.

4. Laube BL (2014) The expanding role of aerosols in systemic drug delivery, gene therapy and vaccination: an update. Transl Respir Med 2: 3.

5. Labiris NR, Dolovich MB (2003) Pulmonary drug delivery. Part I: physiological factors affecting therapeutic effectiveness of aerosolized medications. Br J Clin Pharmacol 56: 588-599.

6. Pleasants RA, Hess DR (2018) Aerosol Delivery Devices for Obstructive Lung Diseases. Respir Care 63: 708-733.

7. Hess D, Fisher D, Peterfreund RA, Kacmarek RM (1995) The delivery of aerosolized steroids from MDIs with nozzle extensions: quantitative laboratory evaluation of a method to improve aerosol delivery to intubated patients. Intensive Care Med 21: 945-951.

8. Godara N, Godara R, Khullar M (2011) Impact of inhalation therapy on oral health. Lung India 28: 272-275.

9. Bousfiha B, Ghazzar FZ, Slaoui B, Ladner J, Hamza M (2016) Oral health in asthmatic children using inhaled therapy. Study in the UTH of Casablanca. Odontostomatol Trop 39: 49-56.

10. Zar HJ, Liebenberg M, Weinberg EG, Binns HJ, Mann MD (1998) The efficacy of alternative spacer devices for delivery of aerosol therapy to children with asthma. Ann Trop Paediatr 18: 75-79.

11. Janssens HM, Tiddens HA (2006) Aerosol therapy: the special needs of young children. Paediatr Respir Rev 7: S83-S85.

12. Lin CL, Tawhai MH, McLennan G, Hoffman EA (2007) Characteristics of the turbulent laryngeal jet and its effect on airflow in the human intra-thoracic airways. Respir Physiol Neurobiol 157: 295-309.

13. Zar HJ, Weinberg EG, Binns HJ, Gallie F, Mann MD (2000) Lung deposition of aerosol--a comparison of different spacers. Arch Dis Child 82: 495-498.

14. Rodriguez-Martinez CE, Sossa-Briceno MP, Castro-Rodriguez JA (2012) Comparison of the bronchodilating effects of albuterol delivered by valved vs. non-valved spacers in pediatric asthma. Pediatr Allergy Immunol 23: 629-635.

15. Waite L, Fine J, Veres G, Szabo G (2009) A velocity driven lumpedparameter model of mitral valve blood flow - biomed 2009. Biomed Sci Instrum 45: 401-406.

16. Oliveira RF, Teixeira SF, Silva LF, Teixeira JC, Antunes H (2012) Development of new spacer device geometry: a CFD study (part I). Comput Methods Biomech Biomed Engin 15: 825-833.

17. Saeed H, Harb HS, Madney YM, Abdelrahim MEA (2021) Aerosol delivery via noninvasive ventilation: role of models and bioanalysis. Ann Transl Med 9: 589.

18. ElHansy MHE, Boules ME, El Essawy AFM, Al-Kholy MB, Abdelrahman MM, et al. (2017) Inhaled salbutamol dose delivered by jet nebulizer, vibrating mesh nebulizer and metered dose inhaler with spacer during invasive mechanical ventilation. Pulm Pharmacol Ther 45: 159-163.

19. West GB, Brown JH, Enquist BJ (1999) The fourth dimension of life: fractal geometry and allometric scaling of organisms. Science 284 : 1677-1679.

20. Radhakrishnan H, Kassinos S (2009) CFD modeling of turbulent flow and particle deposition in human lungs. Conf Proc IEEE Eng Med Biol Soc 2009: 2867-2870.
21. Vanaki SM, Holmes D, Suara K, Jayathilake PG, Brown R (2020) Transport and fate of inhaled particles after deposition onto the airway surface liquid: A 3D numerical study. Comput Biol Med 117: 103595.

22. Longest PW, Bass K, Dutta R, Rani V, Thomas ML, et al. (2019) Use of computational fluid dynamics deposition modeling in respiratory drug delivery. Expert Opin Drug Deliv 16: 7-26.

23. Jiang J, Messner S, Kelm JM, van Herwijnen M, Jennen DGJ, et al. (2019) Human 3D multicellular microtissues: An upgraded model for the in vitro mechanistic investigation of inflammation-associated drug toxicity. Toxicol Lett 312: 34-44.

24. Weinhart M, Hocke A, Hippenstiel S, Kurreck J, Hedtrich S (2019) 3D organ models-Revolution in pharmacological research? Pharmacol Res 139: 446-451.

25. Zscheppang K, Berg J, Hedtrich S, Verheyen L, Wagner DE, et al. (2018) Human Pulmonary 3D Models For Translational Research. Biotechnol J 13: 1700341.

26. Moharamzadeh K, Colley H, Murdoch C, Hearnden V, Chai WL, et al. (2012) Tissue- engineered oral mucosa. J Dent Res 91: 642-650.

27. Boda B, Benaoudia S, Huang S, Bonfante R, Wiszniewski L, et al. (2018) Antiviral drug screening by assessing epithelial functions and innate immune responses in human 3D airway epithelium model. Antiviral Res 156: 72-79.

28. Chen YX, Xie GC, Pan D, Du YR, Pang LL, et al. (2018) Threedimensional Culture of Human Airway Epithelium in Matrigel for Evaluation of Human Rhinovirus $\mathrm{C}$ and Bocavirus Infections. Biomed Environ Sci 31:136-145.

29. Bishop E, Haswell L, Adamson J, Costigan S, Thorne D, et al. (2019) An approach to testing undiluted e-cigarette aerosol in vitro using 3D reconstituted human airway epithelium. Toxicol in vitro 54: 391401.

30. De Servi B, Ranzini F, Pique N (2017) Protective barrier properties of Rhinosectan ${ }^{\circledR}$ spray (containing xyloglucan) on an organotypic 3D airway tissue model (MucilAir): results of an in vitro study. Allergy Asthma Clin Immunol 13: 37

31. Fytianos K, Chortarea S, Rodriguez-Lorenzo L, Blank F, von Garnier C, et al. (2017) Aerosol Delivery of Functionalized Gold Nanoparticles Target and Activate Dendritic Cells in a 3D Lung Cellular Model. ACS Nano 11: 375-383.

32. Walenga RL, Tian G, Longest PW (2013) Development of characteristic upper tracheobronchial airway models for testing pharmaceutical aerosol delivery. J Biomech Eng 135: 91010.

33. Nicola M, Soliman YMA, Hussein R, Saeed H, Abdelrahim M (2020) Comparison between traditional and nontraditional add-on devices used with pressurised metered-dose inhalers. ERJ Open Res 6: 00073-2020.

34. Ehtezazi T, Saleem I, Shrubb I, Allanson DR, Jenkinson ID, et al. (2010) The interaction between the oropharyngeal geometry and aerosols via pressurised metered dose inhalers. Pharm Res 27: 175-186.

35. Yao K, Goto K, Nishimura A, Shimazu R, Tachikawa S, et al. (2019) A Formula for Estimating the Appropriate Tube Depth for Intubation. Anesth Prog 66: 8-13.

36. Oh S, Bang S, Kwon W, Shim J (2016) Patient-specific depth of endotracheal intubation-from anthropometry to the Touch and Read Method. Pak J Med Sci 32: 1234-1239.

37. Miura T (2008) Modeling lung branching morphogenesis. Curr Top Dev Biol 81: 291-310. 